\title{
The Ninety-Nine Percent: Focusing on the Patient to Reduce Readmissions
}

\author{
Karen E. Joynt, MD, MPH', 2,3 \\ 'Division of Cardiovascular Medicine, Department of Medicine, Brigham and Women's Hospital, Boston, MA, USA; ${ }^{2}$ Department of Health \\ Policy and Management, Harvard School of Public Health, Boston, MA, USA; ${ }^{3}$ Cardiology Service, Medical Service, VA Boston Healthcare \\ System, Boston, MA, USA.
}

J Gen Intern Med 29(4):556-7

DOI: $10.1007 / \mathrm{s} 11606-013-2732-6$

(c) Society of General Internal Medicine 2013

I $\mathrm{n}$ this issue of the Journal of General Internal Medicine, Singh and colleagues have identified a new $99 \%$-that is, the amount of variation in readmission rates that is NOT explained by differences in performance between hospitals. ${ }^{1}$ This is an interesting and important study, and the authors should be commended for addressing this issue in a rigorous and objective manner. In this analysis of readmissions in Texas hospitals, $99 \%$ of the total variation in risk of readmission was explained by factors outside the hospital. Further, even of the $1 \%$ of variation that was attributable to the hospital, over half was due to differences in patient characteristics, leaving measured hospital characteristics and provider characteristics holding only $0.4 \%$ of total variation.

This study may inadvertently create more questions than it answers. If $99 \%$ of the difference in a patient's risk of readmission is unexplained by hospitals, then what on earth determines why some hospitals in this study had allcondition readmission rates of $15.9 \%$, while others have readmission rates of almost $23 \%$ ? Perhaps even more importantly, given these findings, what can we do to reduce readmissions and improve patient care?

It may be tempting to throw our hands in the air and give up. If there is little variability between hospitals, and if hospital characteristics and inpatient provider characteristics don't matter, then surely hospitals' and providers' efforts to improve post-discharge follow-up, to focus on and prioritize care coordination, to facilitate communication between inpatient and outpatient providers, and to explicitly ask and address our patients' specific barriers to compliance with medical recommendations are all wasted.
I would in fact argue the exact opposite. This study actually has important lessons for both clinical leaders and policymakers not on why we should give up, but on where we should shift our focus in future efforts to improve patient care. Few would disagree that we can do better-we can do better in discharge planning, we can do better in care coordination, and we can certainly do better in improving access to high-quality outpatient care. But what this study suggests is that it's really all about the patients.

We must work harder to meet patients where they are. Prior research has shown that key factors such as lacking a usual source of care, lacking social support, or having a disability can have a profound impact on readmission risk. $^{2-4}$ We know that patients who are poor, uninsured, or underinsured have higher readmission rates, ${ }^{5}$ and we know that patients with depression have higher readmission rates. ${ }^{6}$ While less well quantified, certainly issues like access to stable housing, transportation, and clinics that can accommodate patients who may work night shifts or other off-hours jobs must impact readmission rates. $^{7}$ Each patient will have a specific and unique set of barriers, and each patient will need a different set of solutions to overcome them. Expecting that hospitals are the only-or even the most appropriate-point of care to address these chronic, complex issues is unrealistic. While hospitals are admittedly a logical target for readmission penalties, owing to their large financial stake in the problem and their central relationship to hospitalization, they are not a logical target to come up with the solutions.

The study by Singh et al. should be great encouragement for providers who are not part of the hospital to join, and in many cases lead, the conversation - the very providers who are actually best equipped to provide real, lasting solutions to these problems. Who better to know the hospitalization triggers for a particular patient than his or her primary care

Published online January 18, 2014 
physician, or the nurse practitioner that has been managing his end-stage heart failure for many years? Who better to prevent hospitalization than the social worker who understands the barriers a single working mother faces in accessing high-quality, timely primary care outside an emergency department? Who better to design an intervention to reduce readmissions than the outpatient care coordinator who helps arrange home services for a fiercely independent but disabled senior living alone? Who better to simplify the overwhelming and inconsistent medication regimen for a patient prescribed 15 medications daily, all on different dosing schedules than a pharmacist? Currently, many of the most successful interventions to reduce readmissions are run not by hospitals, but by nurses, and social workers, and care coordinators, and pharmacists ${ }^{8-10}$ - and we should be recognizing and building upon these successes.

This study should also give pause to policymakers. As we move our delivery system and payment models more toward accountable care organizations and bundled payments, policymakers should recognize that readmissions are only part of the story. To truly incentivize innovation in the type of complex, multidisciplinary outpatient care and management that can keep patients out of the hospital, we must shift our focus to developing creative solutions to prevent unnecessary admissions rather than focus on readmissions. This would more closely couple health metrics with patients' goals, which are generally to stay healthy and out of the hospital when feasible, and to be admitted to the hospital for more advanced care when required - independent of a 30-day post-hospitalization measurement window. The outpatient and inpatient settings should have shared responsibility for keeping patients healthy and for treating them appropriately when their health is threatened.

Reducing readmissions is an important goal, but one that has proven to be elusive thus far. This study begins to point us in a different direction to seek the answers-and suggests that we should follow the $99 \%$ and start to look outside our hospital walls to find meaningful, durable solutions for our most vulnerable patients.

Corresponding Author: Karen E. Joynt, MD, MPH; Division of Cardiovascular Medicine, Department of Medicine, Brigham and Women's Hospital, Boston, MA, USA (e-mail: kjoynt@partners.org).

\section{REFERENCES}

1. Singh S, Lin Y-L, Kuo Y-F, Nattinger A, Goodwin J. Variation in the risk of readmission among hospitals: contribution of patient, hospital and inpatient provider. J Gen Intern Med. 2013; doi: 10.1007/s11606-0132723-7.

2. Arbaje AI, Wolff JL, Yu $\mathbf{3}$, Powe NR, Anderson GF, Boult C. Postdischarge environmental and socioeconomic factors and the likelihood of early hospital readmission among community-dwelling Medicare beneficiaries. Gerontologist. 2008;48(4):495-504.

3. Calvillo-King L, Arnold D, Eubank KJ, et al. Impact of social factors on risk of readmission or mortality in pneumonia and heart failure: systematic review. J Gen Intern Med. 2013;28(2):269-282.

4. Kangovi S, Grande D, Meehan P, Mitra N, Shannon R, Long JA. Perceptions of readmitted patients on the transition from hospital to home. J Hosp Med. 2012;7(9):709-712.

5. Allen LA, Smoyer Tomic KE, Smith DM, Wilson KL, Agodoa I. Rates and predictors of 30-day readmission among commercially insured and Medicaid-enrolled patients hospitalized with systolic heart failure. Circ Heart Fail. 2012;5(6):672-679.

6. Jiang W, Alexander J, Christopher E, et al. Relationship of depression to increased risk of mortality and rehospitalization in patients with congestive heart failure. Arch Intern Med. 2001;161(15):1849-1856.

7. Kangovi S, Barg FK, Carter T, Long JA, Shannon R, Grande D. Understanding why patients of low socioeconomic status prefer hospitals over ambulatory care. Health Aff (Millwood). 2013;32(7): 1196-1203.

8. Naylor MD, Brooten D, Campbell R, et al. Comprehensive discharge planning and home follow-up of hospitalized elders: a randomized clinical trial. Jama. 1999;281(7):613-620.

9. Gil M, Mikaitis DK, Shier G, Johnson TJ, Sims S. Impact of a combined pharmacist and social worker program to reduce hospital readmissions. J Manag Care Pharm. 2013;19(7):558-563.

10. Coleman EA, Parry C, Chalmers S, Min SJ. The care transitions intervention: results of a randomized controlled trial. Arch Intern Med. 2006;166(17): 1822-1828. 